CUMQ/HEP 148

\title{
Production of Single Heavy Charged Leptons at a Linear Collider
}

\author{
Erin De Pree* and Marc Shent \\ Particle Theory Group, Department of Physics, \\ College of William and Mary, Williamsburg, VA 2318\%, USA \\ Ismail Turan \\ Department of Physics, Concordia University, \\ 7141 Sherbrooke Street West, Montreal, Quebec, CANADA H4B 1R6
}

(Dated: November 7, 2018)

\begin{abstract}
A sequential fourth generation of quarks and leptons is allowed by precision electroweak constraints if the mass splitting between the heavy quarks is between 50 and $80 \mathrm{GeV}$. Although heavy quarks can be easily detected at the LHC, it is very difficult to detect a sequential heavy charged lepton, $L$, due to large backgrounds. Should the $L$ mass be above $250 \mathrm{GeV}$, it can not be pairproduced at a $500 \mathrm{GeV}$ ILC. We calculate the cross section for the one-loop process $e^{+} e^{-} \rightarrow L \tau$. Although the cross section is small, it may be detectable. We also consider contributions from the two Higgs doublet model and the Randall-Sundrum model, in which case the cross section can be substantially higher.
\end{abstract}

PACS numbers:

Keywords: Heavy Leptons, 2HDM, Randall-Sundrum

*ekdepr@wm.edu

mtsher@wm.edu

ituran@physics.concordia.ca 


\section{INTRODUCTION}

One of the simplest extensions of the Standard Model is the addition of a new, sequential generation of fermions. Interest in this fourth generation has waxed and waned over the years. After precision measurements of the $\mathrm{Z}$ width showed that there are precisely three weakly interacting neutrinos [1], it became clear that the neutrino mass of a fourth generation would have to exceed $45 \mathrm{GeV}$ and interest faded.

During the 90's there was intensive study of the phenomenology of additional quarks and leptons which were not sequential [2, 3]. Many grand unified theories have additional fermions, such as vectorlike isosinglet quarks and leptons, additional vectorlike states arise in gauge-mediated supersymmetry breaking models, and many additional models contain mirrorlike fermions. These models are still of interest, but they do not require sequential fermions (although they can accommodate them).

Interest in a sequential fourth generation faded further with studies of precision electroweak constraints. The recent Particle Data Group analysis claimed that "An extra generation of ordinary fermions is excluded at the $99.999 \%$ CL on the basis of the S parameter alone" [1]. However, this analysis assumes a mass-degenerate fourth generation. Since one of the most striking features of the mass spectrum of the first three generations is the wide range of masses, such an assumption may not be justifiable.

Analyses of the effects of a non-degenerate sequential fourth generation originally focused on the case where the neutrino mass was of $O(50) \mathrm{GeV}$ [5, 6, 7] and used 2001 electroweak data. A comprehensive analysis of the current status of precision electroweak fits and a fourth generation was recently carried out by Kribs, Plehn, Spannowsky and Tait[8]. They noted that the constraints on the oblique parameters from combined electroweak data have been determined by both the LEP Electroweak Working Group [9] and the Particle Data

Group [1]. The two groups used somewhat different datasets and differ by roughly one standard deviation (see Ref. [8] for a detailed discussion of the differences). Kribs et al. used the LEP Electroweak Working Group results, and found that a substantial region of fourth-generation parameter space is in agreement with all experimental constraints. In this region of parameter space, the mass splitting between the $U$ and $D$ quarks is between 50 and $80 \mathrm{GeV}$. Bounds on the mass splitting between the charged lepton, $L$, and the neutrino, $N$, are less constrained since one considers both Dirac and Majorana neutrino masses [10]. 
Thus, we see that precision electroweak data do not exclude a sequential fourth generation. U and D quark production at the Large Hadron Collider (LHC) will be relatively easy to detect. However, the heavy charged lepton, $L$, will be substantially more difficult to detect, primarily due to large backgrounds. Early LHC and SSC studies [11, 12] made the assumption of a massless fourth generation neutrino, and still concluded that detecting a heavy lepton with a mass greater than $250 \mathrm{GeV}$ might not be possible. Calculations of heavy lepton production (with a heavy neutrino) exist [13], but do not include any discussion of signatures or backgrounds. Therefore, it is likely that a heavy charged lepton with a mass greater than $250 \mathrm{GeV}$ will not be detected at the LHC.

At the International Linear Collider (ILC), heavy leptons can be easily produced and detected up to the kinematic limit. However, the initial stage of the ILC will probably be at a center of mass energy of $500 \mathrm{GeV}$, in which case pair production of heavy leptons with masses above $250 \mathrm{GeV}$ will not be possible. The only possible production mechanism would be through single $L$ production, in association with a lighter Standard Model charged lepton. Since mixing between $L$ and $\mu$ or $e$ is expected to be small, we will focus on the process $e^{+} e^{-} \rightarrow L \tau$, which can occur through a nonzero $\theta_{34}$ mixing angle. Although single production of heavy charged leptons has been studied before [2, 3], all of these studies considered vectorlike or mirrorlike leptons, and we know of no calculations of this process with a heavy neutrino at a linear collider. An analysis of sequential heavy charged leptons in $Z$-decays [14] ignored the mass of the heavy neutrino.

In the next section, we present the relevant diagrams in the sequential standard model and calculate the cross section as a function of the $N$ and $L$ masses. Section 3 considers the cross section in the two Higgs doublet model and in the Randall-Sundrum model. Finally, in Section 4, we discuss detection possibilities and present our conclusions.

\section{SEQUENTIAL STANDARD MODEL}

A single charged heavy lepton can only be produced if the fourth generation mixes with the lighter generations. Bounds on the mixing angle $\theta_{34}$ arise from observation of universality in $\tau$ decays; a nonzero mixing angle would multiple the rate by $\cos ^{2} \theta_{34}$. This was analyzed by Swain and Taylor [15] who found a model-independent bound of $\sin ^{2} \theta_{34}<0.007$. This is a particularly interesting value. If one has a Fritzsch-type $2 \times 2$ mass matrix for the $\tau$ and 
$L$, one might expect $\sin ^{2} \theta_{34}$ to be approximately $m_{\tau} / m_{L}$, which gives 0.007 for an $L$ mass of $250 \mathrm{GeV}$. We will assume this value of the mixing angle in our numerical results, and can easily scale the cross section for smaller mixing angles.

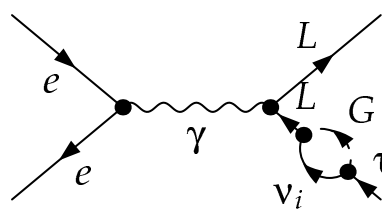

1

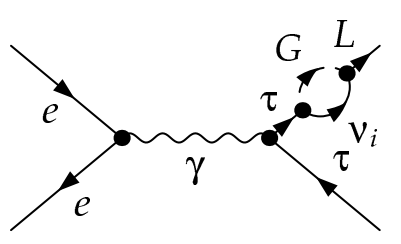

5

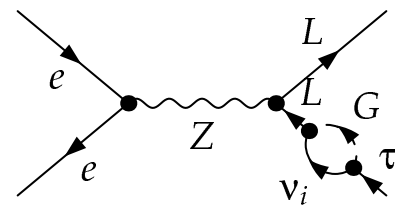

2

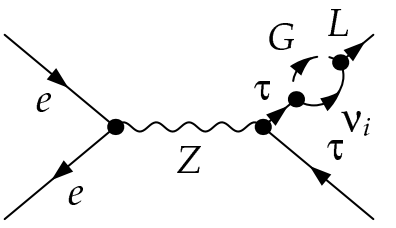

6

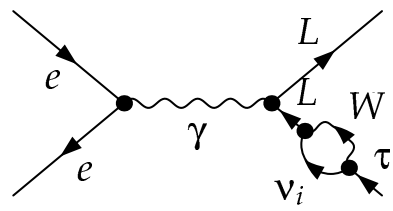

3

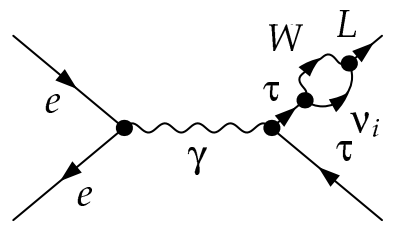

7

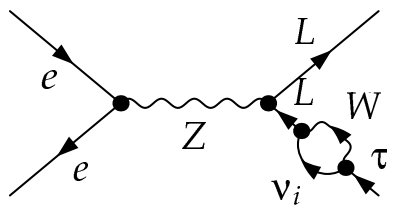

4

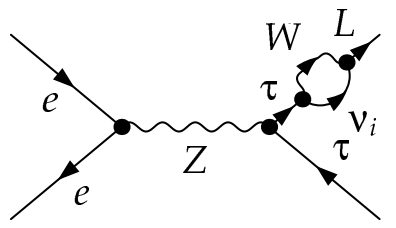

8

FIG. 1: The leading order self energy contributions to the $e^{+} e^{-} \rightarrow L \bar{\tau}$ process in the sequential SM. The Feynman gauge is assumed and the light electron-Higgs couplings are neglected.

The diagrams are listed in Figures 1-3 and grouped as the self energy, vertex and box type contributions, respectively. We use the 't-Hooft-Feynman gauge throughout, and thus charged Goldstone bosons, $G$, must be included. Note that the electron-Higgs couplings are neglected due to small Yukawa couplings. The internal neutrino lines get a contribution from each of the four neutrinos, and thus each diagram is proportional to $V_{4 i}^{*} V_{i 3}$. When summing over the four neutrinos, parts of the matrix elements that are independent of the neutrino mass will cancel by unitarity of the 4-D CKM-like matrix. This causes the ultraviolet divergences to cancel in the sum over neutrinos.

The calculation of the cross section is performed by using the FeynArts, FormCalc, and LoopTools packages [17]. We first patched the SM and 2HDM model files of the FeynArts package by introducing the fourth generation leptons and their interactions. Then, the numerical analysis was carried out in Fortran with the help of FormCalc and LoopTools. The cancellation of the ultraviolet and infrared divergences has been checked numerically and the expected cancellation was confirmed. In addition, as a separate check the expected null result for the cross section due to unitarity of the mixing matrix $V_{i j}$ was also tested 


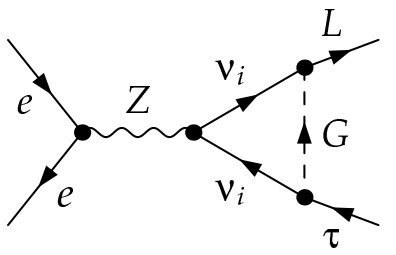

1

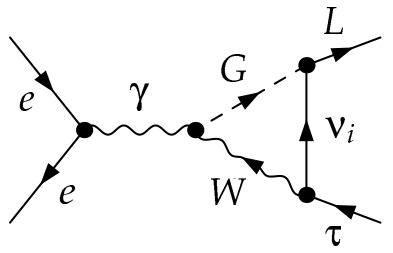

5

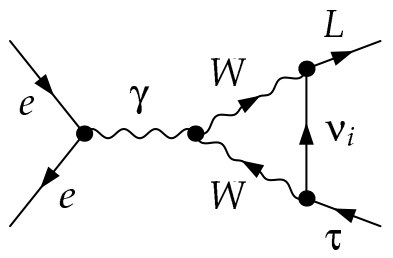

9

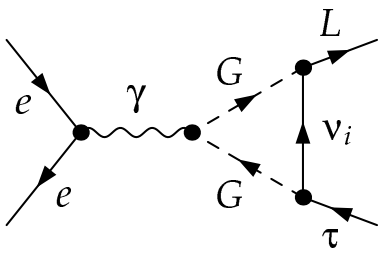

2

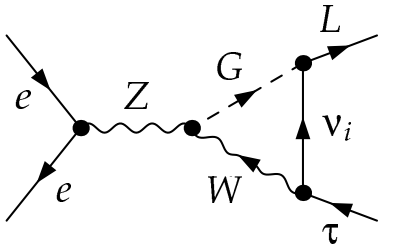

6

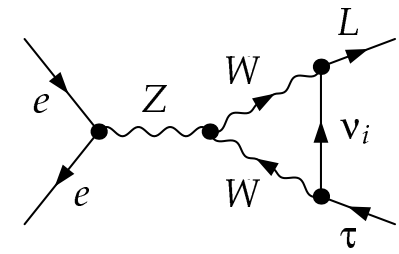

10

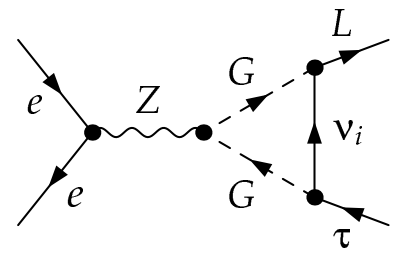

3

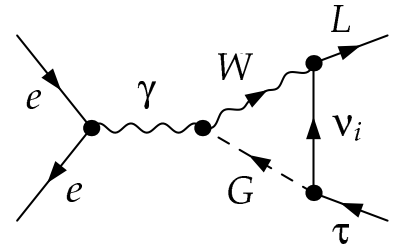

7

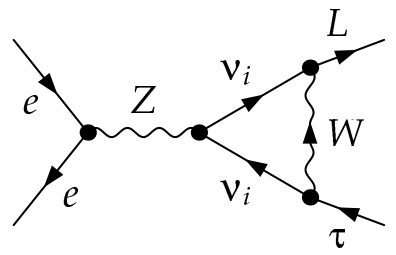

4

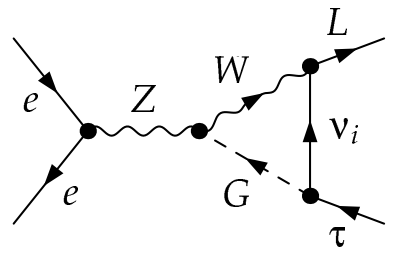

8

FIG. 2: The leading order vertex contributions to the $e^{+} e^{-} \rightarrow L \bar{\tau}$ process in the sequential SM.The 't-Hooft-Feynman gauge is assumed and the light electron-Higgs couplings are neglected.

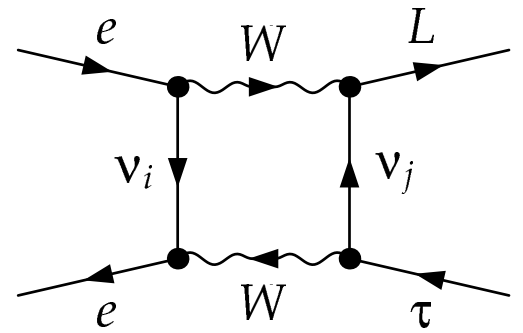

FIG. 3: The leading order box contributions to the $e^{+} e^{-} \rightarrow L \bar{\tau}$ process in the sequential SM. The 't-Hooft-Feynman gauge is assumed and the light electron-Higgs couplings are neglected.

numerically by setting the heavy neutrino mass $m_{N}$ to zero. Note that the same technique is applied for the calculation in the $2 \mathrm{HDM}$, presented in the next section.

The results are plotted in Figure 4 for neutrino masses of 300, 400 and $500 \mathrm{GeV}$. We see that, for $\sin ^{2} \theta_{34}=0.007$, cross sections of the order of a few attobarns can be expected. We can also show that as the neutrino mass increases, the cross section grows rapidly, reaching 


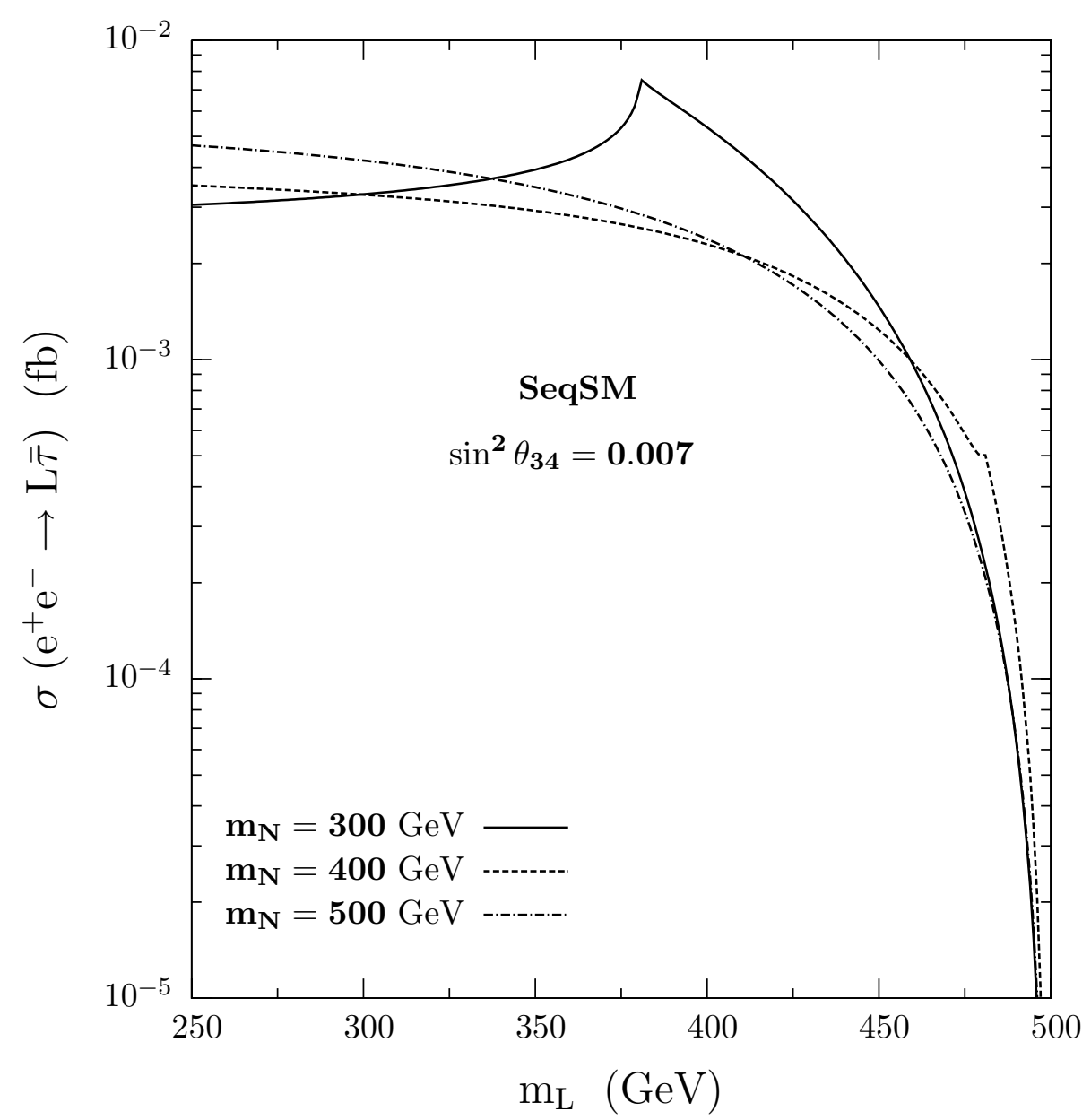

FIG. 4: The total cross section of $e^{+} e^{-} \rightarrow L \bar{\tau}$ as a function of the heavy lepton mass $m_{L}$ for $\sqrt{\mathrm{s}}=500 \mathrm{GeV}$ and various heavy neutrino masses in an unpolarized electron-positron beam within the Sequential SM framework.

500 attobarns at $M_{N}=2000 \mathrm{GeV}$. This is not surprising since the theory is chiral. Of course, the cross section scales with $V_{34}^{2}$; the value we have chosen is the maximum allowed from the analysis of Swain and Taylor.

The structure of the curves in Figure 4 can be easily understood. Since the theory is chiral, one expects the cross section to increase as the mass of the heavy neutrino $m_{N}$ increases. However, as seen from Figure 4 that this is not necessarily true for neutrinos in the $300-400 \mathrm{GeV}$ mass range. One can understand, for example, why the the curve for $m_{N}=300 \mathrm{GeV}$ crosses and becomes bigger than the one for $m_{N}=400 \mathrm{GeV}$ and similar behavior occurs between the $m_{N}=400 \mathrm{GeV}$ and $m_{N}=500 \mathrm{GeV}$ curves. This is simply due to the fact that both the $W$ boson and the heavy neutrino $N$ go on-shell in the loop 
if the condition $m_{L} \geq m_{W}+m_{N}$ is kinematically satisfied. When $m_{L}$ is large enough to produce the $W$ and $N$ on-shell, the loop integrals develop imaginary parts, which can be calculated by using the Cutkosky rules, and results in enhancement of the cross section. One can calculate this by cutting through the $W$ boson- $\nu_{i}$ propagators (for $i=4$ ) at the heavy lepton's leg in Figures 1-3. Thus, for example, the peak due this enhancement for the $m_{N}=300 \mathrm{GeV}$ curve occurs at around $m_{N}+m_{W}$ and it shifts to the right for the $m_{N}=400$ GeV curve.

Are these small cross sections detectable? With an integrated luminosity of an inverse attobarn, expected at the ILC's full luminosity for a couple of years, one expects a handful of events. The tau is monochromatic, and is opposite a monochromatic $\mathrm{W}$ and a light neutrino. We know of no backgrounds to this signature, and a complete analysis would be worthwhile.

\section{THE TWO-HIGGS DOUBLET AND RANDALL-SUNDRUM MODELS}

\section{A. The Two-Higgs Doublet Model}

The minimal Standard Model Higgs sector consists of one complex Higgs doublet. One of the simplest and most popular extensions of the Higgs sector is the two-Higgs doublet model (2HDM). By requiring that all fermions of a given electric charge couple to no more than one Higgs doublet [16], one avoids flavor changing neutral currents. This is accomplished with a simple $Z_{2}$ symmetry.

The $2 \mathrm{HDM}$ is an attractive model for several reasons:

- it contains charged Higgs bosons and pseudoscalars

- it adds relatively few new arbitrary parameters

- it allows for spontaneous $\mathrm{CP}$ violation, and can give sufficient baryogenesis

- this structure of the Higgs sector is required in low-energy supersymmetric models

A very detailed discussion of the 2HDM can be found in the Higgs Hunter's Guide [18].

This model has two complex, $Y=1, S U(2)_{L}$ doublet scalar fields $\phi_{1}$ and $\phi_{2}$. The vacuum expectation values of the neutral components of the Higgs doublets are $v_{1}$ and $v_{2}$, 
respectively. It is useful to define

$$
\tan \beta=\frac{v_{2}}{v_{1}}
$$

The physical Higgs fields consist of two neutral scalars, a neutral pseudoscalar and a charged Higgs scalar. In the charged sector, there will be both a Goldstone boson and a physical Higgs state. The charged Higgs is given by

$$
H^{ \pm}=-\phi_{1}^{ \pm} \sin \beta+\phi_{2}^{ \pm} \sin \beta
$$

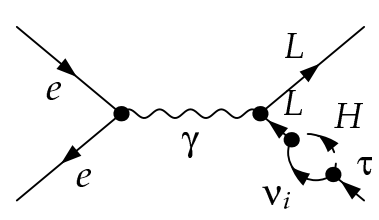

1

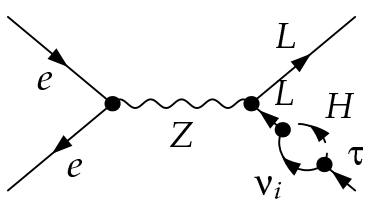

2

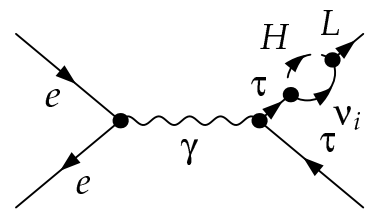

3

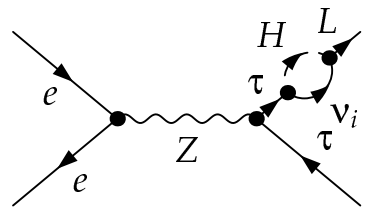

4

FIG. 5: The extra self energy diagrams contributing to $e^{+} e^{-} \rightarrow L \bar{\tau}$ in $2 \mathrm{HDM}$.

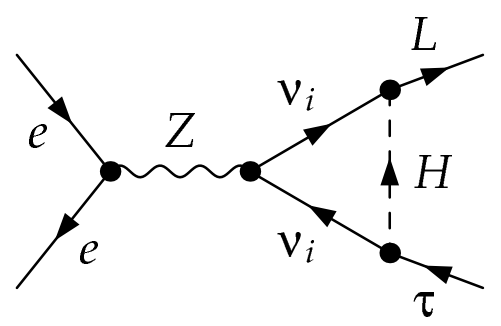

1

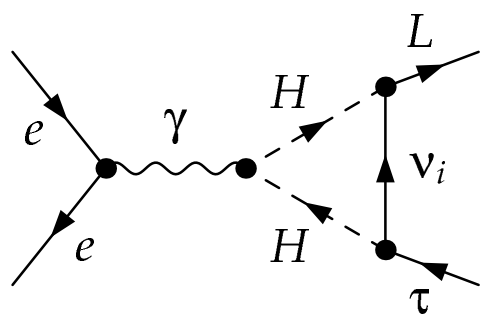

2

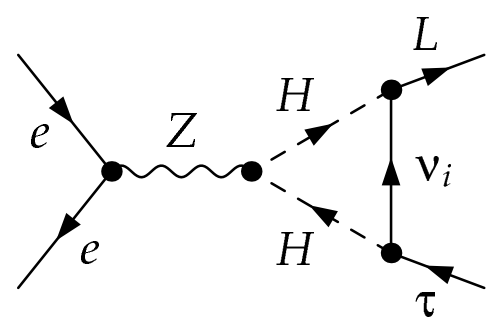

3

FIG. 6: The extra vertex diagrams contributing to $e^{+} e^{-} \rightarrow L \bar{\tau}$ in $2 \mathrm{HDM}$.

For our calculation, the neutral scalars will not contribute. However, the charged Higgs boson will contribute. One simply replaces the charged Goldstone boson $G$ in Figures 1-3 with the charged Higgs boson; these diagrams are shown in Figures 5 and 6 . The only exception is that the $Z W^{\mp} H^{ \pm}$vertex vanishes [18]. There are now two new parameters in the calculation, the mass of the charged Higgs boson and $\tan \beta$.

There are two versions of the 2HDM. In Model I, all of the fermions couple to one of the Higgs doublets; in Model II (which is included in supersymmetric models), the neutral 
leptons couple to one doublet and the charged leptons couple to the other. The relevant Yukawa couplings are

$$
\begin{aligned}
L-N-H^{+}: & \frac{i e}{2 \sqrt{2} m_{W} \sin \theta_{W}}\left[\frac{m_{N}}{\tan \beta}\left(1-\gamma_{5}\right)+m_{L} Y\left(1+\gamma_{5}\right)\right], \\
e_{i}-N-H^{+}: & \frac{-i e \delta_{i, 3} V_{34}}{2 \sqrt{2} m_{W} \sin \theta_{W}}\left[\frac{m_{N}}{\tan \beta}\left(1-\gamma_{5}\right)+m_{e i} Y\left(1+\gamma_{5}\right)\right], \\
L-\nu_{i}-H^{+}: & \frac{i e \delta_{i, 3} V_{34}}{2 \sqrt{2} m_{W} \sin \theta_{W}} m_{L} Y\left(1+\gamma_{5}\right),
\end{aligned}
$$

where $Y=-1 / \tan \beta$ for Model I and $\tan \beta$ for Model II and the vertices for the ordinary lepton - ordinary neutrino $-H^{+}$can be found in the Higgs Hunter's Guide [18].

Constraints from $b \rightarrow s \gamma$ force the mass of the charged Higgs to exceed approximately $200 \mathrm{GeV}$ [19]. $\tan \beta$ and $\cot \beta$ must be less than about 3 so that the charged and neutral lepton Yukawa coupling remain perturbative.
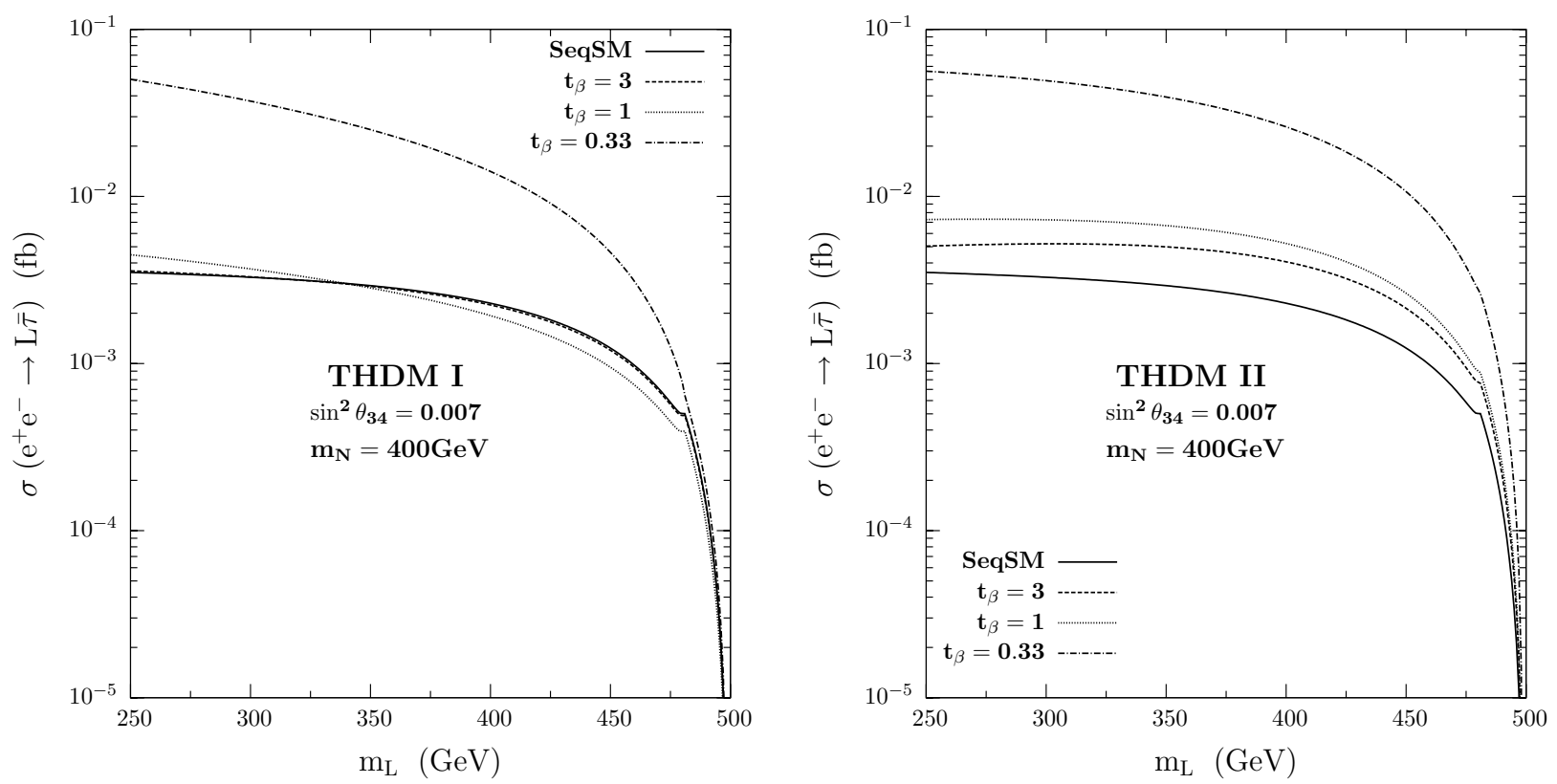

FIG. 7: The total cross section of $e^{+} e^{-} \rightarrow L \bar{\tau}$ as a function of the heavy lepton mass $m_{L}$ for $\sqrt{\mathrm{s}}=500 \mathrm{GeV}$ in an unpolarized electron-positron beam for various $\tan \beta$ values in $2 \mathrm{HDM}$. In both graphs, the heavy neutrino and charged Higgs masses are set $400 \mathrm{GeV}$ and $200 \mathrm{GeV}$, respectively.

The results are presented for Model I and for Model II in Figure 7, We see small changes in the cross section for $1<\tan \beta<3$, but substantial changes for $1<\cot \beta<3$. In both models, the cross section can be enhanced by up to a factor of ten, leading to much easier detection at the ILC. Note that the vertices involving the heavy neutrino scales a $1 / \tan \beta$, and thus the cross section is enhanced if $\tan \beta<1$ as seen from Figure 7 , 


\section{B. The Randall-Sundrum Model}

Another popular extension of the Standard Model is the Randall-Sundrum (RS1) model

[20]. In this model a slice of $A d S_{5}$ space with curvature $k$ is compactified on an $S_{1} / Z_{2}$ orbifold with radius $R$. The fixed points of the orbifold correspond to two 3-branes with opposite tensions. The 4D graviton is localized on the positive tension, or Planck brane, while the Higgs field is localized on the negative tension, or $\mathrm{TeV}$ brane. This model generates a gauge hierarchy given by the warp factor, $e^{-\pi k R}$, and for $k R \simeq 12$ completely solves the hierarchy problem (see [21] for a bound on $k R$ from the Casimir force measurements).

In the original formulation, the model had all of the fermions on the TeV brane. More interesting phenomenology can occur when the Standard Model fermions and gauge bosons can propagate in the bulk [22]. In this case, the profiles of bulk fermion wavefunctions depend on their 5D mass parameters. By choosing the lighter fermions to live near the Planck brane, one can naturally explain the small Yukawa couplings for the light fermions, since their overlap with the TeV-brane localized Higgs boson is exponentially supressed. Thus the model can also explain the flavor hierarchy, since large differences in Yukawa couplings can arise from small differences in the mass parameters. The flavor hierarchy simply becomes a matter of geography in the fifth dimension.

In an interesting series of papers, Agashe, Perez and Soni [23, 24, 25] discussed the phenomenological implications of the flavor structure of these models. They noted that one expects larger flavor changing neutral currents for the heavier generations, thus evading bounds involving light quarks. In particular [25], Agashe, et al. considered top flavor violation at colliders, considering $t \rightarrow c Z$ at the LHC, and $e^{+} e^{-} \rightarrow t \bar{c}$ at the ILC. Clearly, a similar process could lead to $e^{+} e^{-} \rightarrow L \tau$ as well, if a fourth generation exists. The mechanism is caused by the fact that the couplings of the fermions to the gauge boson Kaluza-Klein $(\mathrm{KK})$ modes are not universal due to the different profiles for the fermions, and mixing between the gauge KK modes and the gauge bosons leads to flavor violating couplings of the $Z$. We refer the reader to Ref. [25] for details.

One can simply carry over the calculation of $e^{+} e^{-} \rightarrow t \bar{c}$ in Ref. [25] to this model. There is, however, one crucial difference. Since the couplings of the left-handed $b$ quark to the $Z$ are measured to an accuracy of less than one percent and the $b$ quark is in a doublet with the left-handed top, one can not put the left-handed top and bottom too close to the TeV brane. 
The right-handed top, however, can be close to the TeV brane. Thus the top flavor violation is predominantly right-handed. In the four generation case, there are no such restrictions, therefore the $L$ flavor violation is relatively unconstrained. For definiteness, we choose the same magnitude for the left- and right-handed flavor violation, and set the coefficient of the $\bar{L} \gamma_{\mu} \tau Z^{\mu}$ term to be the same as that of the $\bar{t}_{R} \gamma_{\mu} c_{R} Z^{\mu}$ term in the Agashe, et al. analysis [25]. This is not unreasonable, since $m_{c} / m_{t} \sim m_{\tau} / m_{L}$ indicates that similar mixing angles may be expected.

Using this flavor violating coupling, one can find the total cross section for $e^{+} e^{-} \rightarrow$ $L \tau$. The result depends as well on the KK scale. It has been shown [26] that a custodial $S U(2)$ symmetry in the bulk can allow the KK gauge boson mass to be as low as $3 \mathrm{TeV}$, and perhaps somewhat lower if a modest fine-tuning is allowed, without conflicting with precision electroweak results. Rather than calculate the interference with the Standard Model diagrams, we simply look at the RS model effects in isolation. This is because the uncertainty in the flavor-violating couplings preclude precise calculations. We find that if $M_{K K}$ is $1 \mathrm{TeV}$, then the cross section varies from 1.0 to 0.5 femtobarns as the $L$ mass varies from $250 \mathrm{GeV}$ to $350 \mathrm{GeV}$, and scales as $1 / M_{K K}^{4}$. Thus, we see a significant enhancement of the cross section in the KK mass range of $1-3 \mathrm{TeV}$. One should keep in mind that the KK gauge bosons, if they exist, will be discovered at the LHC long before the ILC is constructed.

\section{DETECTION AND CONCLUSIONS}

There are two possible decay modes for the $L$. It can decay into $N W$, or into $\nu_{\tau} W$. Of course, if the $N$ is heavier only the latter decay is possible. Regardless, there will be substantial missing energy in the decay.

For the $e^{+} e^{-} \rightarrow L \tau$ process detection should be extremely straightforward, since the $\tau$ is monochromatic. For an $L$ mass of $300 \mathrm{GeV}$, the $\tau$ energy is $160 \mathrm{GeV}$, leading to a decay length, $\gamma c t$, of 0.8 centimeters. This is comparable to the size of the inner vertex detector at the ILC.

In a wide region of the mass-mixing angle plane, the $L$ will decay into a $\nu_{\tau}$ and a $W$. This would seem to give a clear signature, with a monochromatic $\tau$, a monochromatic $W$ and missing energy. The primary background will be from $\tau$ pairs, where one of the $\tau$ 's is misidentified. A detailed Monte Carlo analysis is beyond the scope of this paper, but if the 
background can be eliminated, then a few events will suffice to discover the $L$.

In the Standard Model case, we have found that there will be a few events produced at the ILC, and the question of whether or not the $L$ can be detected depends on the details of the detector and Monte Carlo simulations.

We then considered contributions from the charged Higgs boson of a 2HDM, as well as flavor changing effects in the Randall-Sundrum model. In both cases, there are regions of parameter space in which the cross section is substantially higher, leading to straightforward detection at the ILC.

Long before the ILC is built, the LHC will have determined whether or not a fourth generation exists. If it does exist, then detection of the charged heavy lepton at the LHC will be very difficult and perhaps impossible. At the ILC, if the mass of the heavy lepton is more than half $\sqrt{s}$, pair production will be impossible, and the process calculated in this paper may be the only mechanism for detection.

We thank Chris Carone and Josh Erlich for their useful comments, and David Reiss and Thomas Hahn for assistance with the software packages. This work is supported by the National Science Foundation, Grant PHY-0554854. The work of I.T. is supported by the NSERC of Canada under the Grant No. SAP01105354.

[1] W. M. Yao et al. [Particle Data Group], J. Phys. G 33, 1 (2006).

[2] For an extensive discussion and a comprehensive list of references prior to 2000, see P. H. Frampton, P. Q. Hung and M. Sher, Phys. Rept. 330, 263 (2000) arXiv:hep-ph/9903387.

[3] F. del Aguila, M. Perez-Victoria and J. Santiago, JHEP 0009, 011 (2000) arXiv:hep-ph/0007316; J. E. Cieza Montalvo and M. D. Tonasse, Nucl. Phys. B 623, 325 (2002) arXiv:hep-ph/0008196; S. Nie and M. Sher, Phys. Rev. D 63, 053001 (2001) arXiv:hep-ph/0011077]; A. Arhrib and W. S. Hou, Phys. Rev. D 64, 073016 (2001) arXiv:hep-ph/0012027;

[4] K. M. Belotsky and M. Y. Khlopov, Grav. Cosmol. Suppl. 8N1, 112 (2002); H. Ciftci and S. Sultansoy, Mod. Phys. Lett. A 18, 859 (2003) arXiv:hep-ph/0107321; D. Choudhury, T. M. P. Tait and C. E. M. Wagner, Phys. Rev. D 65, 053002 (2002) arXiv:hep-ph/0109097; J. A. Aguilar-Saavedra, Phys. Rev. D 67, 035003 (2003) [Erratum-ibid. D 69, 099901 
(2004)] arXiv:hep-ph/0210112]; A. Arhrib and W. S. Hou, Eur. Phys. J. C 27, 555 (2003) arXiv:hep-ph/0211267]; J. E. Cieza Montalvo and M. D. Tonasse, Phys. Rev. D 67, 075022 (2003) arXiv:hep-ph/0302235; D. E. Morrissey and C. E. M. Wagner, Phys. Rev. D 69, 053001 (2004) arXiv:hep-ph/0308001; M. Y. Khlopov, Pisma Zh. Eksp. Teor. Fiz. 83, 3 (2006) [JETP Lett. 83, 1 (2006)] [arXiv:astro-ph/0511796]; P. Q. Hung, Int. J. Mod. Phys. A 20, 1276 (2005) arXiv:hep-ph/0406257; G. A. Kozlov, A. N. Sisakian, Z. I. Khubua, G. Arabidze, G. Khoriauli and T. Morii, J. Phys. G 30, 1201 (2004); J. A. Aguilar-Saavedra, Phys. Lett. B 625, 234 (2005) [Erratum-ibid. B 633, 792 (2006)] [arXiv:hep-ph/0506187]; A. T. Alan, A. Senol and N. Karagoz, Phys. Lett. B 639, 266 (2006) arXiv:hep-ph/0511199]; R. Mehdiyev, S. Sultansoy, G. Unel and M. Yilmaz, Eur. Phys. J. C 49, 613 (2007) arXiv:hep-ex/0603005]; J. A. Aguilar-Saavedra, PoS TOP2006, 003 (2006) arXiv:hep-ph/0603199; A. Arhrib and W. S. Hou, JHEP 0607, 009 (2006) arXiv:hep-ph/0602035.

[5] H. J. He, N. Polonsky and S. F. Su, Phys. Rev. D 64, 053004 (2001) arXiv:hep-ph/0102144.

[6] M. Maltoni, V. A. Novikov, L. B. Okun, A. N. Rozanov and M. I. Vysotsky, Phys. Lett. B 476, 107 (2000) arXiv:hep-ph/9911535.

[7] V. A. Novikov, L. B. Okun, A. N. Rozanov and M. I. Vysotsky, Phys. Lett. B 529, 111 (2002) arXiv:hep-ph/0111028; V. A. Novikov, L. B. Okun, A. N. Rozanov and M. I. Vysotsky, JETP Lett. 76, 127 (2002) [Pisma Zh. Eksp. Teor. Fiz. 76, 158 (2002)] arXiv:hep-ph/0203132.

[8] G. D. Kribs, T. Plehn, M. Spannowsky and T. M. P. Tait, arXiv:0706.3718 [hep-ph].

[9] J. Alcaraz et al. [ALEPH Collaboration], arXiv:hep-ex/0612034. http://lepewwg.web.cern.ch/LEPEWWG/plots/summer2006/s06_stu_contours.eps

[10] E. Gates and J. Terning, Phys. Rev. Lett. 67, 1840 (1991); B. A. Kniehl and H. G. Kohrs, Phys. Rev. D 48, 225 (1993); B. Holdom, JHEP 0608, 076 (2006) arXiv:hep-ph/0606146.

[11] R. M. Barnett et al., Snowmass DPF Summer Study, 1988, p. 159.

[12] S. S. D. Willenbrock and D. A. Dicus, Phys. Lett. B 156, 429 (1985).

[13] P. H. Frampton, D. Ng, M. Sher and Y. Yuan, Phys. Rev. D 48, 3128 (1993) arXiv:hep-ph/9210270.

[14] J. I. Illana and T. Riemann, Phys. Rev. D 63, 053004 (2001) arXiv:hep-ph/0010193.

[15] J. Swain and L. Taylor, Phys. Rev. D 55, 1 (1997) arXiv:hep-ph/9610242.

[16] S. L. Glashow and S. Weinberg, Phys. Rev. D 15, 1958 (1977).

[17] T. Hahn and M. Perez-Victoria, Comput. Phys. Commun. 118, 153 (1999); T. Hahn, 
Nucl. Phys. Proc. Suppl. 89, 231 (2000); T. Hahn, Comput. Phys. Commun. 140, 418 (2001); T. Hahn, C. Schappacher, Comput. Phys. Commun. 143, 54 (2002); T. Hahn, arXiv:hep-ph/0506201.

[18] J. F. Gunion, H. E. Haber, G. L. Kane and S. Dawson, The Higgs Hunter's Guide, (Perseus, Cambridge, Massachusetts, USA), 1990.

[19] F. Borzumati and A. Djouadi, Phys. Lett. B 549, 170 (2002) arXiv:hep-ph/9806301.

[20] L. Randall and R. Sundrum, Phys. Rev. Lett. 83, 3370 (1999) arXiv:hep-ph/9905221.

[21] M. Frank, I. Turan and L. Ziegler, Phys. Rev. D 76, 015008 (2007) [arXiv:0704.3626 [hep-ph]].

[22] H. Davoudiasl, J. L. Hewett and T. G. Rizzo, Phys. Lett. B 473, 42 (2000) arXiv:hep-ph/9911294; Y. Grossman and M. Neubert, Phys. Lett. B 474, 361 (2000) arXiv:hep-ph/9912408; T. Gherghetta and A. Pomarol, Nucl. Phys. B 586, 141 (2000) arXiv:hep-ph/0003129.

[23] K. Agashe, G. Perez and A. Soni, Phys. Rev. Lett. 93, 201804 arXiv:hep-ph/0406101.

[24] K. Agashe, G. Perez and A. Soni, Phys. Rev. D 71, 016002 (2004) arXiv:hep-ph/0408134.

[25] K. Agashe, G. Perez and A. Soni, Phys. Rev. D 75, 015002 (2007) arXiv:hep-ph/0606293.

[26] K. Agashe, A. Delgado, M. J. May and R. Sundrum, JHEP 0308, 050 (2003) arXiv:hep-ph/0308036. 